\title{
L a muerte fetal y la muerte neonatal tienen origen multifactorial*
}

\author{
LILIA HUIZA ${ }^{1,2}$, PERCY PACORA ${ }^{3}$, MÁXIMO AYALA ${ }^{3}$, YTALA BUZZIO $^{4}$ \\ ${ }^{1}$ Instituto de Patología UNM SM. . ${ }^{2}$ Anatomía Patológica UNFV. ${ }^{3}$ D epartamento de \\ Gineco-O bstetricia UNM SM. ${ }^{4} \mathrm{H}$ ospital Nacional D ocente Madre-Niño San Bartolomé
}

\begin{abstract}
RESUMEN
OBJETIV O: I dentificar los factores etiopatogénicos asociados a la muerte fetal y neonatal de una población de la ciudad de Lima, Perú. LU GA R: Hospital San Bartolomé en Lima, entre 1 de enero de 1996 y 31 de diciembre de 2000. M A TERIA L Y MÉTODOS: Estudio retrospectivo de todos los casos de muerte fetal mayor de 20 semanas de gestación y muerte neonatal antes de los 28 días de nacimiento sometidos a estudio de autopsia y anatomía patológica. RESU LTA DOS: L a prevalencia de muerte fetal fue $1,68 \%(508 / 30125)$ y de muerte neonatal $1,44 \%$ (434/30125). Todas las muertes fetales y $58,8 \%$ (255/434) de las muertes neonatales tuvieron autopsia. De 508 muertes fetales, 92 (18\%) presentaron signos de autólisis, que no permitieron una apropiada identificación de los procesos etiopatogénicos involucrados. $L$ as muertes fetales estuvieron asociadas a prematuridad $(60,5 \%)$, defecto nutricional/ vascular $(78,1 \%)$, privación social $(60,8 \%)$, anomalías congénitas anatómicas $(14,6 \%)$ y respuesta inflamatoria (12,9\%). L a muerte neonatal estuvo asociada a la prematuridad $(71,7 \%)$, privación social $(61,1 \%)$, defecto nutricional/vascular $(59,6 \%)$, anomalía congénita anatómica $(28,6 \%)$, respuesta inflamatoria (20,0\%). CON CL U SION ES: L a muerte fetal es consecuencia de factores patogénicos que interactúan tempranamente con la unidad madre-embrión/feto. La mayoría de los tejidos muertos de los fetos/neonatos muestra manifestaciones de varios factores etiopatogénicos en forma simultánea.
\end{abstract}

Palabras clave: Embarazo de alto riesgo; muerte fetal; neonatología.

\section{ETIOLOGY OF FETAL AND NEONATAL DEATH IS MULTIFACTORIAL SUM MARY}

OBJECTIVE: To identify the etiopathogenic factors associated to both fetal and neonatal deaths in Lima, Peru. MATERIALS A ND M ETHODS: Retrospective study of all the cases of fetal death over 20 weeks gestation and neonatal death before 28 days of life that underwent autopsy and histopathologic study, in order to identify the cause of perinatal death. SETTIN G: Hospital San Bartolomé in Lima, between January 1, 1996 and December 31, 2000. RESULTS: The prevalence of fetal death was $1,68 \%(508 / 30125)$ and neonatal death 1,44\% (434/30125). All fetal deaths and 58,8\% (255/434) of the neonatal deaths underwent autopsy. A mong 508 fetal deaths, $92(18 \%)$ presented signs of autolysis that did not al low appropiate identification of the etiopathogenic process. F etal death was associated to prematurity $(60,5 \%)$, nutricional/vascular defect $(78,1 \%)$, social privation $(60,8 \%)$, congenital anomaly $(14,6 \%)$ and inflammatory response $(12,9 \%)$. N eonatal death was associated to prematurity $(71,7 \%)$, social privation $(61,1 \%)$, nutritional/vascular defect $(59,6 \%)$, congenital anomaly $(28,6 \%)$ and infammatory response $(20,0 \%)$. CONCLUSIONS: F etal death is a consequence of pathogenic factors that interact early with the maternal-embryo/fetus unit. F etal/neonatal death tissue usually show histologic findings of several pathogenic factors simultaneously.

Key words: Pregnancy, high-risk; fetal death; neonatology.

* El resumen del presente trabajo fue publicado en Anales de la Facultad de M edicina, Volumen 63, Suplemento 2002.

Correspondencia:

Dr. Percy Pacora Portella

Hospital Docente Madre Niño "San Bartolomé"

Av. Alfonso U garte 825. Lima 1, Perú

E-mail: kalebna@terra.com.pe 


\section{INTRODUCCIÓN}

L a enfermedad y la muerte del feto y el recién nacido están asociadas a diversos factores relacionados a los genes y al medio ambiente. A sí, se ha descrito que la muerte fetal tardía se asocia a la restricción del crecimiento fetal, mujeres con talla menor de $156 \mathrm{~cm}$, embarazos múltiples e hipertensión arterial ( $\left.{ }^{1}\right)$.

En embarazos de peso normal al nacer, el riesgo de muerte fetal se asocia a la edad de la madre, el hábito de fumar, embarazos múltiples, e hipertensión arterial $(1,2)$.

Los riesgos de complicaciones placentarias aumentan con la edad de la madre, ya que se ha visto cambios vasculares degenerativos en las arterias uterinas y miometriales en mujeres en edad reproductiva $\left({ }^{2,3}\right)$.

D ebido a que existe una escasez de información de las causas histológicas de muerte fetal y neonatal en nuestro medio, se realizó esta investigación, con la finalidad de identificar las causas patológicas asociadas a la muerte fetal y neonatal en una población de mujeres gestantes en Lima.

\section{MATERIAL Y MÉTODOS}

Se realizó un estudio descriptivo retrospectivo de todos los casos de muerte fetal mayor de 20 semanas de gestación y de las muertes neonatales menor de 28 días de vida que tuvieron estudio anatomo-patológico entre el 1 de enero de 1996 y el 31 de diciembre de 2000. Se obtuvo, además, la información demográfica materna y perinatal del sistema informático perinatal (SIP) del Servicio de Obstetricia del Hospital San Bartolomé (SIP) durante el mismo periodo de estudio.

$\checkmark$ arias características maternas fueron analizadas, tales como la edad materna, peso previo al embarazo, talla materna, estado civil, grado de instrucción, hábito de fumar y sexo fetal, a fin de reconocer las características maternas y perinatales que identificaba a la mayoría de las muertes fetales y neonatales.

Se definió como estado de privación social a la paciente que presentaba alguno de las siguientes factores: 1) ausencia de control prenatal, 2) menos de 5 años de educación formal y 3) estado civil soltera. El grado de privación social se graduó de cero (0) a tres (3). Si la madre tenía 1) control prenatal , 2) más de 5 años de educación formal y 3) era casada o conviviente, la paciente recibía un puntaje " 0 ". Se agregó un punto por cada uno de los factores ausentes. A sí, la paciente con ausencia de estos tres factores sociales obtenía un puntaje de "3" y se definía como estado de privación social severo.

Se empleó estadística descriptiva y análisis univariado de cada variable materna y perinatal en los casos de muerte fetal y neonatal, empleando la tabla de 2 por 2 (prueba exacta de Fisher). Se calculó el odds ratio y el intervalo de confianza al 95\% (IC 95\%) para cada variable materna y perinatal, empleando el programa estadístico SPSS 10,0.

T omando en cuenta los hallazgos histológicos y clínicos maternoperinatales, las causas patogénicas de las muertes fetal es/neonatales fueron clasificadas en: 1) prematuridad (gestación $<37$ semanas), 2) anomalías congénitas anatómicas, 3) respuesta inflamatoria (corioamnionitis, funisitis, villitis, sepsis fetal o neonatal), 4) defecto nutricional/vascular (anemia, hemorragia, isquemia, trombosis, traumatismos, estenosis) y 5) privación social.

\section{RESULTADOS}

La prevalencia de muerte fetal fue $1,68 \%$ $(508 / 30125)$ y de muerte neonatal $1,44 \%$ (434/ 30125), en el periodo en estudio.

La característica materna de la población de gestantes atendidas en el hospital San Bartolomé durante ese periodo aparece en la Tabla 1: 14, 4\% era menor de 20 años, 14\% madre soltera, 0,8\% presentaba privación social severa, $0,8 \%$ IM C 
Tabla 1.- Característica materna en 30125 gestaciones atendidas entre enero 1996 y diciembre 2000. Hospital San Bartolomé. Lima, Perú.

\begin{tabular}{lcl}
\hline Edad (promedio \pm DE), años. & $25,9 \pm 6,1$ & (rango: $10-49$ ) \\
Peso pregestacional (promedio \pm DE), kg. & $55,6 \pm 7,9$ & (rango: $30-99$ ) \\
Talla (promedio \pm DE), cm & $155,3 \pm 5,9$ & (rango: $12-701-199$ ) \\
Índice de masa corporal (promedio $\pm \mathrm{DE}$ ), $\mathrm{kg} / \mathrm{m}^{2}$ & $23,0 \pm 3,1$ & \\
Años de educación & $4,3 \pm 1,1$ & \\
Privación social & $51,2 \%$ & \\
Multípara* & $46,2 \%$ & \\
Edad mayor de 30 años & $27,0 \%$ & \\
Menos de cinco años de educación & $27,8 \%$ & \\
Un aborto previo & $23,5 \%$ & \\
IMC mayor de $24,9 \mathrm{~kg} / \mathrm{m}^{2}$ & $30,8 \%$ & \\
IMC menor de $20,0 \mathrm{~kg} / \mathrm{m}^{2}$ & $13,1 \%$ & \\
Ausencia de control prenatal & $20,9 \%$ &
\end{tabular}

* Un hijo previo nacido.

mayor de $34,9 \% \mathrm{~kg} / \mathrm{m}^{2}$ y $0,3 \%$ tenía el hábito de fumar.

Los factores clínicos asociados a la muerte fetal en forma significativa aparecen en la Tabla 2. También se asociaron a la muerte fetal las gestantes con diversas patologías (OR: 2,61 , IC $95 \%: 2,11-3,24)$, particularmente de tipo infecciosa (OR: 2,59, IC 95\%: 1,41-4,75), en los que se tuvo que emplear antibiótico (OR: 2,29, IC $95 \%: 1,25-4,19)$ y se requirió hospitalizar a la madre antes del parto (OR: 2,0, IC 95\%: 1,452,92 ), la historia de natimuerto (OR: 2,45, IC 95\%: 1,40-4,28), el embarazo múltiple (OR: 2,35, IC 95\%: 1,49-3,70), el IMC > 34,9 kg/ mt2 (OR: 2,18, IC 95\%: 1,02-4,65), la privación social (OR: 1,58, IC 95\%: 1,30-1,92), el aborto previo (OR: 1,35, IC 95\%: 1,10-1,67),

Tabla 2.- Factores de riesgo asociado de muerte fetal en 528 gestantes que presentaron muerte fetal, comparado con 29515 gestantes que no tuvieron muerte fetal.

\begin{tabular}{lccc}
\hline & OR & IC $95 \%$ & Valor de p \\
\hline Muerte materna & 46,77 & $14,34-152,49$ & $<0,001$ \\
Peso bajo al nacer & 29,14 & $23,74-35,76$ & $<0,001$ \\
Nacimiento pretérmino & 24,00 & $19,51-29,53$ & $<0,001$ \\
Anormalidad anatómica neonatal & 16,55 & $5,55-49,29$ & $<0,001$ \\
Sangrado vaginal en segundo trimestre & 14,59 & $7,48-28,45$ & $<0,001$ \\
Sangrado vaginal en el embarazo & 10,10 & $7,53-13,55$ & $<0,001$ \\
Sangrado vaginal en el tercer trimestre & 10,09 & $7,24-14,05$ & $<0,001$ \\
Hipertensión previa & 8,09 & $3,24-20,21$ & $<0,001$ \\
Feto pequeño para edad gestacional & 7,34 & $5,72-9,43$ & $<0,001$ \\
Eclampsia & 5,71 & $1,78-18,34$ & $<0,05$ \\
Defecto congénito & 5,08 & $3,08-8,36$ & $<0,001$ \\
Feto podálico/ transverso & 4,49 & $3,46-5,82$ & $<0,001$ \\
Hemorragia puerperal & 3,54 & $2,06-6,08$ & $<0,001$ \\
Ausencia de control prenatal & 3,17 & $2,60-3,85$ & $<0,001$ \\
Amenaza de parto pretérmino & 2,84 & $1,76-4,58$ & $<0,001$ \\
\hline
\end{tabular}


la edad mayor de 30 años (OR: 1,29, IC 95\%: 1,05-1,59) y la multiparidad (OR : 1,29, IC 95\%: $1,06-1,56)$.

Los factores clínicos que disminuían el riesgo de muerte fetal fueron: Ia alfabetización de la madre (OR: 0,18, IC 95\%: 0,07-0,46), el alumbramiento completo (OR: 0,17, IC 95\%: $0,14-0,21)$, la ausencia de patología materna (OR: 0,50, IC 95\%: 0,41-0,62) y el sexo fetal varón (OR: 0,79, IC 95\%: 0,65-0,96).

La frecuencia de las características clínicas de las 508 muertes fetales fueron: nacimiento pretérmino $346(68,1 \%)$, peso bajo al nacer $332(65,4 \%)$, privación social $309(60,8 \%)$, un hijo previo $261(51,4 \%)$, ausencia de control prenatal $207(40,7 \%)$, edad mayor de 30 años $162(31,9 \%)$, aborto anterior $147(28,9 \%)$, patologías maternas varias $138(27,1 \%)$, madre hospitalizada previo al parto $131(25,8 \%)$, feto pequeño $113(22,3 \%)$, feto podálico/ transverso $82(16,2 \%)$, sangrado vaginal en el embarazo 67 $(13,2 \%)$, sangrado vaginal en el tercer trimestre $49(9,7 \%)$, madre hospitalizada antes del parto $43(8,3 \%)$, embarazo múltiple $22(4,3 \%)$, el defecto congénito $21(4,2 \%)$, la amenaza de parto pretérmino $21(4,2 \%)$, hemorragia puerperal 16,3 $(3,2 \%)$ y un natimuerto previo $15(3,0 \%)$.

Se pudo estudiar histológicamente 508 muertes fetales y $58,8 \%(255 / 434)$ de las muertes neonatales, porque los padres consintieron la autopsia. De 508 muertes fetales, 92 (18\%) presentaron signos de autólisis que no permitieron realizar el reconocimiento histológico de las posibles causas de muerte.

La Tabla 3 muestra el resultado del estudio por histología de los 416 (82\%) fetos que pudieron ser estudiados adecuadamente. Las causas reconocibles por histología asociadas a la muerte fetal fueron: prematuridad $60,5 \%(252 / 416)$, defecto nutricional/vascular 78,1\% (325/416), anomalías congéni tas anatómicas 14,6\% (61/416) y la respuesta inflamatoria 12,9\% (54/416).

Los factores clínicos asociados a la muerte neonatal aparecen en la Tabla 4. También estuvieron asociadas a la muerte neonatal la historia
Tabla 3.- Hallazgos histológicos en 416 muertes fetales.

\begin{tabular}{lrc}
\hline \multicolumn{1}{c}{ Causa } & $\mathrm{n}$ & $\%$ \\
\hline Prematuridad & 252 & 60,5 \\
Anomalías congénitas anatómicas & 61 & 14,6 \\
Defecto nutricional y/o vascular & 325 & 78,1 \\
- Peso menor de 2500 g & 260 & 62,5 \\
- Hipoxia tisular & 65 & 15,6 \\
Respuesta inflamatoria & 54 & 12,9 \\
\hline
\end{tabular}

Hipoxia tisular: hemorragia pulmonar y anemia.

de hijo con peso bajo (OR: 3,35, IC 95\%: 1,36$8,24)$, ausencia de control prenatal (OR: 3,06 IC 95\%: 2,56-3,67), parto por cesárea (OR: 2,79, IC 95\%: 2,34-3,33), anemia crónica (OR : 2,69, IC $95 \%: 2,18-3,32)$, ruptura prematura de membranas (OR: 2,69, IC 95\%: 2,18-3,32), madre hospitalizada antes del parto (OR : 2,64, IC 95\%: 1,96-3,54), empleo de antibióticos (OR: 2,38, IC 95\%: 1,36-4,16), infección puerperal (OR: $2,36$, IC $95 \%: 1,45-3,86)$, otras patologías maternas (OR: 2,17, IC 95\%: 1,76-2,67), ruptura de membranas > 12 horas (OR: 2,16, IC 95\%: 1,69-2,75), otras infecciones (OR: 1,99 , IC $95 \%$ : $1,06-3,76)$, ruptura prematura de membranas $>$ 6 horas (OR: 1,90, IC 95\%: 1,50-2,39), prediabetes-diabetes (OR 1,83 IC 95\% 1, 12-2,98), privación social(OR: 1,66, IC 95\%: 1,39-1,99), infección urinaria (OR: 1,56, IC 95\%: 1,241,95), aborto previo (OR: 1,52, IC 95\%: 1,251,83 ), preeclampsia-eclampsia (OR: 1,44, IC 95\%: 1,16-1,80), edad mayor de 30 años (OR: 1,33, IC 95\%: 1,10-1,60), neonato varón (OR: 1,33 , IC 95\%: 1, 12-1,59). L a ausencia de enfermedad o complicación materna disminuía significativamente el riesgo de muerte neonatal (OR: 0,24, IC 95\%: 0, 19-0,31).

$L$ a frecuencia de los factores de riesgo asociados a la muerte neonatal fueron: parto pretérmino 321 (74\%), privación social 265 $(61,1 \%)$, anormalidad anatómica 247 (56,9\%), neonato varón $250(57,6 \%)$, parto por cesárea $204(47,0 \%)$, ausencia de control prenatal 168 $(38,7 \%)$, edad mayor de 30 años $139(32 \%)$, aborto previo $135(31,1 \%)$, peso bajo al nacer / 
Tabla 4.- Factores de riesgo de muerte neonatal en 434 gestantes con muerte neonatal, comparado con 29691 gestantes que no tuvieron muerte neonatal.

\begin{tabular}{lccc}
\hline & OR & IC 95\% & Valor de p \\
\hline Peso bajo al nacer & 58,24 & $47,14-71,96$ & $<0,001$ \\
Anormalidad anatómica & 37,04 & $30,51-44,95$ & $<0,001$ \\
Nacimiento pretérmino & 35,12 & $28,71-42,96$ & $<0,001$ \\
Muerte materna & 10,77 & $1,34-86,32$ & $<0,001$ \\
Amenaza de parto pretérmino & 8,70 & $6,52-11,62$ & $<0,001$ \\
Sangrado vaginal en tercer trimestre & 7,13 & $4,97-10,22$ & $<0,001$ \\
Recién nacido pequeño para edad gestacional & 6,60 & $5,42-8,03$ & $<0,001$ \\
Sangrado vaginal en segundo trimestre & 6,56 & $2,63-16,37$ & $<0,001$ \\
Sangrado vaginal en el embarazo & 6,37 & $4,58-8,86$ & $<0,001$ \\
Embarazo múltiple & 5,83 & $4,34-7,84$ & $<0,001$ \\
Feto podálico/transverso & 5,31 & $4,22-6,69$ & $<0,001$ \\
Privación social severa & 3,82 & $2,12-6,85$ & $<0,001$ \\
Restricción del crecimiento fetal & 3,64 & $2,62-5,06$ & $<0,001$ \\
Sífilis materna & 3,59 & $1,31-9,81$ & $<0,05$ \\
\hline
\end{tabular}

Privación social: ausencia de control prenatal, menos de 5 años de educación formal o ser madre soltera

pequeño para la edad $131(30,2 \%)$, patologías maternas varias $102(23,5 \%)$, ruptura prematura de membranas $102(22,8 \%)$, preeclampsiaeclampsia $85(19,6 \%)$, anemia crónica 84 $(19,3 \%)$, infección urinaria $80(18,5 \%)$, feto podálico/transverso $80(18,5 \%)$, ruptura de membranas mayor de 6 horas $75(17,4 \%)$, ruptura de membrana mayor de 12 horas $69(15,8 \%)$, amenaza de parto pretérmino $48(11,0 \%)$, embarazo múltiple $43(10,0 \%)$, madre hospitalizada antes del parto $43(10,0 \%)$, sangrado vaginal en el embarazo $35(8,1 \%)$, restricción de crecimiento fetal $34(7,8 \%)$, sangrado vaginal en el tercer trimestre $29(6,7 \%)$, prediabetes-diabetes 15 $(3,5 \%)$ e infección puerperal $15(3,5 \%)$.

La Tabla 5 muestra las causas reconocibles por histología asociadas a las muertes neonatales, destacando la prematuridad, defecto nutricional / vascular, anomalías congénitas y la respuesta inflamatoria.

\section{DISCUSIÓN}

Esta investigación demuestra que la muerte fetal y la muerte neonatal obedecen a varios factores patogénicos que intervienen en forma si-
Tabla 5.- Hallazgos histológicos en 255 muertes neonatales.

\begin{tabular}{lrc}
\hline \multicolumn{1}{c}{ Causa } & $\mathrm{n}$ & $\%$ \\
\hline Prematuridad & 183 & 71,7 \\
Defecto nutricional/vascular & 152 & 59,6 \\
- Peso bajo al nacer & 77 & 30,1 \\
- Hipoxia tisular & 75 & 29,4 \\
Anomalía congénita anatómica & 73 & 28,6 \\
Infección & 51 & 20,0 \\
\hline
\end{tabular}

multánea. Así, más de $50 \%$ de las muertes fetales estuvieron asociadas al nacimiento pretérmino, la desnutrición fetal (peso bajo al nacer), privación social en la madre y presencia de un hijo previo. La inmadurez de los órganos fetales (prematuridad) y los defectos nutricionales/ vasculares ocurrieron en más de $60 \%$ de las muertes fetales ( $T$ abla 3 ).

Debido a que el hábito de fumar no es frecuente en nuestra población estudiada $(0,3 \%)$, no pudimos encontrar algún ef ecto de este hábito sobre la muerte fetal, como ha sido reportado en el extranjero $(1,2)$.

L a salud de la madre está estrechamente ligada a la salud del feto. A sí, la edad de la ma- 
dre, la obesidad materna marcada (IM C > 34, 9 $\mathrm{kg} / \mathrm{m}^{2}$ ), la historia previa de natimuerto, el aborto previo y las complicaciones maternas -tales como sangrado vaginal, hipertensión arterial previa, eclampsia, hemorragia puerperal-, las patologías variadas maternas e infecciones que requieren el empleo de antibióticos e internamiento de la madre antes del parto, estuvieron asociadas significativamente a la muerte fetal.

En la práctica, es muy difícil separar la salud física del ser humano de la salud mental y del medio social donde vive. Nosotros hemos encontrado que la privación social de la madre se asoció con un riesgo significativamente aumentado de muerte fetal. Es más, $40 \%$ de las muertes fetales ocurrió en mujeres sin control prenatal. Pensamos que la ausencia de control prenatal no sólo comprende la ausencia de atención profesional, sino que además es la consecuencia de: 1) la falta de un entorno social favorable, que le permita a la mujer gestante acudir en busca de ayuda profesional, 2) la falta de fé de la mujer y su familia hacia los profesionales de la salud, y 3) la falta de una actitud mental positiva (proactiva) de la madre hacia el bienestar del hijo. Este último argumento lo sustentamos porque, en más de la mitad de los casos de muertes fetales $(51,3 \%)$, las mujeres habían tenido un hijo previo de un parto anterior, $31,8 \%$ era mayor de 30 años, $29 \%$ había abortado anteriormente y $60,8 \%$ se encontraba con algún grado de privación social. Indudablemente, el entorno social de la mujer influye en la salud mental y ambos factores (social-emocional) son difíciles de separar en cada persona. En una investigación anterior, nuestro grupo encontró que la privación social y/o afectiva en la madre repercute negativamente en el resultado materno y perinatal $\left(^{5}\right)$.

Similarmente, Guildea y col $\left({ }^{6}\right)$ han encontrado que la muerte fetal y neonatal se incrementan en $53 \%$ en áreas muy deprimidas socialmente, comparado con los lugares menos deprimidos. Estos investigadores encontraron que la muerte infantil aumentaba en $120 \%$ en los lugares más deprimidos socialmente, comparado con los menos deprimidos; la muerte neonatal aumentaba en $41 \%$ y la muerte neonatal $20 \%$. Por otro lado, el análisis de nuestro estudio encontró que la alfabetización de la madre (OR: 0,18) disminuía en forma significativa el riesgo de muerte fetal. Estos resultados nos señalan que el medio más eficaz para disminuir la muerte fetal consiste en mejorar la situación social de la mujer.

Si bien la estimación del estado nutricional en casos de muerte fetal es difícil de realizar debido a que la edad gestacional y el peso fetal al nacer se establecen al momento del nacimiento y este hecho conduciría a una sobreestimación de la edad gestacional y al peso bajo al nacer, debido a que el feto muerto puede perder peso antes del nacimiento, la autopsia perinatal del feto o recién nacido nos proporciona valiosa información sobre las causas de muerte. Si la autopsia es realizada en forma estandarizada por un patólogo perinatal $\left({ }^{7-10}\right)$, la información que se obtiene tiene un gran efecto en la forma cómo los padres son aconsejados en los riesgos para futuros embarazos y permite conocer la opinión de los padres sobre el diagnóstico prenatal temprano en futuros embarazos. Gran parte de esta valiosa información no puede ser obtenida de otra forma (11). A sí, aún la ultrasonografía en manos expertas tiene una sensibilidad de $97 \%$ para detección de fetos con anomalías congénitas, comparado con la autopsia perinatal. Sin embargo, cuando se considera todas las anomalías anatómicas encontradas durante la autopsia, la sensibilidad de la ultrasonografía en la detección de las anomalías congénitas mayores es $75 \%$ y la sensibilidad en la detección de las anomalías menores 18\% (12). Por lo tanto, la autopsia del feto o recién nacido brinda valiosa información para los padres sobre el riesgo de futuros embarazos en caso de muertes fetal es. $V$ arios estudios han demostrado que de $14 \%$ a $46 \%$ de las autopsias brindan nueva información y, en al rededor de $10 \%$, el diagnóstico definitivo de la causa de muerte es cambiado (7-12,13-15).

En nuestro estudio fue necesario conocer la historia clínica materna o perinatal en más de la mitad de los casos, para comprender la etiopatogenia de la muerte perinatal. R evisando toda la información clínico-patológica, encontramos que en más de $60 \%$ de los casos de muer- 
te fetal existía un factor de privación social materna y desnutrición fetal. Por otro lado, la muerte neonatal estaba asociada, también en más de $60 \%$, a la privación social de la madre y en cerca de $30 \%$ a factores anatómicos (anomalías congénitas) y desnutrición neonatal.

Realizamos el análisis de regresión logística múltiple de multivariado de las muertes fetales y neonatales de las gestaciones simples, donde se ingreso al modelo la edad materna, el índice de masa corporal y el grado de privación social (variables continuas) y la historia previa de un aborto, el parto de un hijo previo, el examen físico normal del recién nacido, la anomalía congénita anatómica, el peso bajo al nacer, nacimiento pretérmino y sangrado vaginal durante el embarazo (variables categóricas), para determinar factores de riesgos independiente. Encontramos que el hallazgo macroscópico de anormalidad fetal (feto anormal), el sangrado vaginal durante el embarazo y el peso bajo era factores independientes de riesgo fetal. Por otro lado, Ia privación social fue el factor independiente de la muerte neonatal (datos no mostrados). Estos hallazgos nos señalan que el efecto de la privación social se manifiesta en forma independiente en la muerte neonatal; en cambio, la muerte fetal es una consecuencia de varias variables que ocurren durante el embarazo.

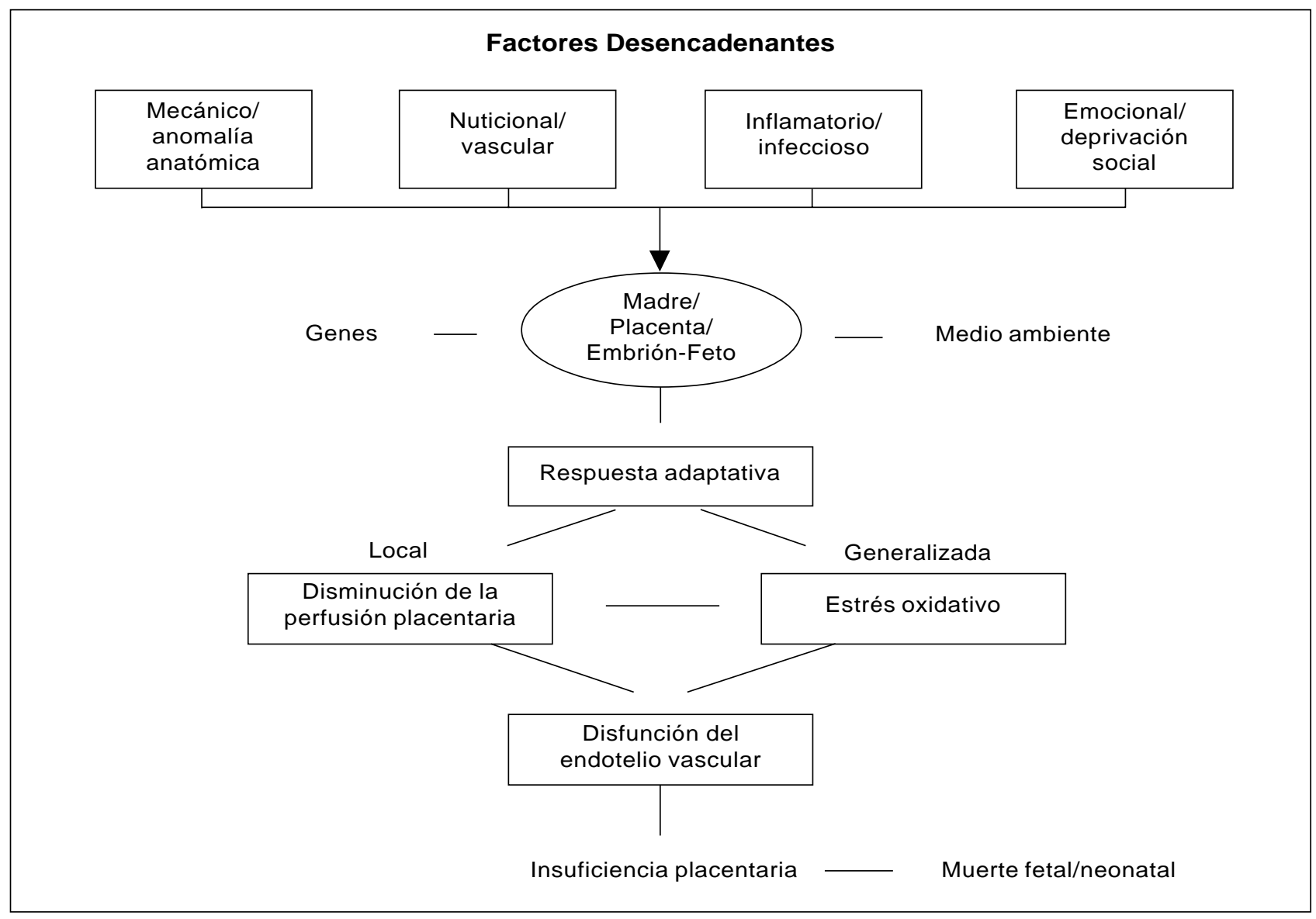

Figura. Interacción madre-placenta-feto en la muerte perinatal. Los factores desencadenantes mecánicos, nutricionales, inflamatorios y emocionales interactúan sobre el organismo vivo materno-embrión/feto, el cual responde en forma local: disminuyendo la perfusión placentaria, y en forma generaliza: desarrollando el estrés oxidativo. Ambos mecanismos dañan el endotelio vascular materno/ fetal, dando el cuadro clínico de insuficiencia placentaria y la consecuente muerte fetal o neonatal si no se interviene oportunamente. 
En vista de estos hallazgos, proponemos que la causa de muerte fetal/neonatal es de origen multifactorial, ya que un feto con anomalías congénitas anatómicas de origen genético puede asociarse con factores de perfusión placentaria pobre, restricción del crecimiento fetal, hipoxia e inflamación. Por otro lado, un feto con restricción del crecimiento debido a factores de depresión emocional materno, puede asociarse con infección e hipoxia ( $\left.{ }^{4}\right)$.

Los hallazgos histológicos de las muertes neonatales en este estudio mostraron que diversos factores etiopatogenicos intervinieron en forma simultánea, tales como defecto nutricional/ vascular, anomalía congénita, prematuridad, respuesta inflamatoria y privación social.

En conclusión, los hallazgos de este estudio demuestran que la muerte fetal/neonatal ocurre por factores patogénicos de origen multifactorial. L a mayor parte de las muertes fetales/neonatales ocurren por la participación de factores patogénicos que intervienen tempranamente en la unidad madre-concepto. De manera que la salud física, mental y social de la madre está estrechamente ligada al bienestar del hijo antes de nacer y la salud del hijo después del nacimiento. La muerte fetal/neonatal se manifiesta por alteraciones tisulares de tipo nutricional/ vascular, defecto funcional por inmadurez de los órganos (prematuridad), respuesta inflamatoria (infecciones), anomalías anatómicas y privación social/afectiva. Por lo tanto, la muerte fetal y la muerte neonatal es de origen multifactorial, tal como puede apreciarse en la Figura.

\section{AGRADE CIMIENTO}

Este estudio pudo realizarse gracias al financiamiento de Educación, Conocimiento y Salud, S.A.C.

\section{BIBLIOGRAFÍA}

1. Cnattingius $\mathbf{S}, \mathbf{H}$ aglund $\mathbf{B}, \mathbf{K}$ ramer $\mathbf{M S}$. Differences in late fetal death rates in association with determinants of small for gestational age fetuses: population based cohort study. BMJ 1998; 316: 1483-7.

2. Cnattingius $\mathbf{S}, \mathbf{L}$ arsson $\mathbf{E}, \mathbf{W}$ einer $\mathbf{E}$. Uterine-arterial changes with age and smoking. Int J Feto-M aternal Med 1990; 3: 15-8.

3. Naeye $\mathbf{R} \mathbf{L}$. M aternal age, obstetric complications, and the outcome of pregnancy. Obstet Gynecol 1983; 61: 210-6.

4. Williams MC, $\mathbf{O}^{\prime}$ Brien $\mathbf{F}$, Nelson $\mathbf{N}$, Spellacy $\mathbf{N}$. $\mathrm{H}$ istologic chorioamnionitis is associated with fetal growth restriction in term and preterm infants. A $\mathrm{m} J$ Obstet Gynecol 2000; 183: 1094-9.

5. Pacora P, Tello T, Capcha E, Esquivel L, Ayala M, H uiza L. La privación social y/o afectiva en la madre repercute negativamente en el resultado materno y perinatal. A nal Fac M ed U NM SM 2002; 63 (Suppl): 47.

6. Guildea ZE S, Fone DL, Dunstan FD, Sibert J R, C artlidge PHT. Social deprivation and the causes of stillbirth and infant mortality. A rch Dis Child 2001; 84: 307-10.

7. Cartlidge PH, Dawson AT, Stewart J H, V ujanic GM. $V$ alue and quality of perinatal and infant postmortem examinations: cohort analysis of 400 consecutive deaths. BM J 1995; 310: 155-8.

8. Thornton CM, $\mathbf{O}^{\prime}$ Hara M D. A regional audit of perinatal autopsies in N orthern Ireland. Br J Obstet Gynaecol 1998; 105: 18-23.

9. Vujanic GM, Cartlidge PH, Stewart J H, Dawson AJ. Perinatal and infant postmortem examinations: how well are we doing? J Clin Pathol 1995; 48: 998-1001.

10. Wright $\mathbf{C}$, Cameron $\mathbf{H}, \mathbf{L}$ amb W. A study of the quality of perinatal autopsy in the former Northern Region. $\mathrm{Br}$ J Obstet Gynaecol 1998; 105: 24-8.

11. Cox P, Scott R. Perinatal autopsy in 2001. A rch Dis Child 2001; 84: 457-8.

12. Y eo L, G uzman ER, Shen-Schwarz S, Walters C, V intzileos AM . V alue of a complete sonographic survey in detecting fetal abnormalities: correlation with perinatal autopsy. J Ultrasound M ed 2002; 21(5): 501-10.

13. Shen-Schwarz S, Neish C, Hill L M. A ntenatal ultrasound for fetal anomalies: importance of perinatal autopsy. Pediatr Pathol 1989; 9: 1-9.

14. Porter HJ, Keeling J W, Dawson AJ. V alue of perinatal necropsy examination. J Clin Pathol 1987; 40: 180-4.

15. Gould S, Keeling J, Thornton C M. Perinatal pathology. Confidential enquiry into stillbirths and deaths in infancy, 6th annual report. London: $M$ aternal and $C$ hild Health Research Consortium, 1999; 51-8. 